Meta

Journal des traducteurs

Translators' Journal

\title{
L’université face au développement
}

\section{Jean-Claude Gémar}

Volume 35, numéro 2, juin 1990

URI : https://id.erudit.org/iderudit/002866ar

DOI : https://doi.org/10.7202/002866ar

Aller au sommaire du numéro

Éditeur(s)

Les Presses de l'Université de Montréal

ISSN

0026-0452 (imprimé)

1492-1421 (numérique)

Découvrir la revue

Citer cette note

Gémar, J.-C. (1990). L’université face au développement. Meta, 35(2), 457-459.

https://doi.org/10.7202/002866ar

Ce document est protégé par la loi sur le droit d'auteur. L'utilisation des services d’Érudit (y compris la reproduction) est assujettie à sa politique d'utilisation que vous pouvez consulter en ligne.

https://apropos.erudit.org/fr/usagers/politique-dutilisation/
Cet article est diffusé et préservé par Érudit.

Érudit est un consortium interuniversitaire sans but lucratif composé de l’Université de Montréal, l’Université Laval et l’Université du Québec à Montréal. Il a pour mission la promotion et la valorisation de la recherche. https://www.erudit.org/fr/ 


\section{L'UNIVERSITÉ FACE AU DÉVELOPPEMENT}

La IV ${ }^{\mathrm{e}}$ Rencontre mondiale des départements d'études françaises et du dialogue des cultures s'est tenue à New Delhi du 15 au 19 décembre 1988 sous l'égide de l'AUPELF et de l'Unitersité Jawaharlal Nehru, sur le thème Université et développement.

Cette Rencontre a rassemblé 250 participants venant de 60 pays différents.

\section{Sous-thème I}

Le sous-thème I a porté sur «le français, langue de communication et de culture scientifique».

Une des lignes directrices des ateliers a été constituée par une réflexion sur l'enseignement des langues de spécialité.

Un débat s'est instauré entre les participants autour des trois grands thèmes suivants :

a) D'une part, est-il bien dans la mission de l'Université de se pencher sur l'enseignement d'un ou des français de spécialité ?

Il apparaît qu'il faille, par raison ou par choix délibéré, en arriver à cette démarche, pour laquelle l'Université et ses enseignants sont souvent mal préparés. Encore convient-il de cerner très précisément les cadres, la motivation et les champs d'application de ces langues.

Ainsi, une première recommandation est-elle faite à l'AUPELF d'encourager l'élaboration d'une typologie des domaines considérés, des situations d'apprentissage liées aux besoins et aux paramètres locaux et des didactiques appropriées. Les stratégies d'apprentissage dépendent de la combinatoire de ces différents éléments.

b) D'autre part, au travers de communications très diversifiées, l'inventaire des matériels d'enseignement existants a fait très largement apparaître la primauté des créations locales sur l'utilisation ou l'adaptation de manuels ou de méthodes commercialisés. Ainsi, la réflexion, l'élaboration, la mise en œuvre et l'évaluation de ces types d'enseignement de spécialité sont-elles fortement individualisées.

Les participants ont souhaité qu'à l'initiative de l'AUPELF, il soit fait place à l'institutionnalisation d'un échange de ces expériences, pour éventuellement une meilleure adéquation des besoins et de leurs réponses, mais aussi pour une nécessaire confrontation interrégionale et interdisciplinaire. Celle-ci est la condition nécessaire, car motivante et vivifiante, à une meilleure prise en compte, par les enseignants et les autorités entièrement ou partiellement en place, de ce type de formation. À ce propos, il est demandé à l'AUPELF d'être l'élément moteur d'une dynamique de formation des enseignants à ces formes didactiques qui ne correspondent pas aux disciplines traditionnelles.

De ce fait même, au-delà de la motivation, l'efficacité de l'apprentissage des langues de spécialité se mesurera à l'aune du savoir-faire communicatif, même si l'on doit négliger quelque peu, et c'est parfois préférable, l'étude du domaine de spécialité luimême.

La pratique de la traduction, et nécessairement dans ces domaines, ne peut ni ne doit être négligée, pour autant.

Si une fois encore, comme à l'occasion d'autres rencontres ou colloques régionaux, il a été demandé de porter une attention toute particulière à la formation des traducteurs, un débat de fond a permis de dégager ces lignes de force, pouvant faire office de recommandations.

D'une part, si le savoir-faire est bien entendu essentiel, il n'en demeure pas moins qu'il est indispensable de prendre très largement en compte le savoir-être.

D'autre part, et cette recommandation dépasse par essence le cadre de la réflexion sur la traduction, la distinction doit être faite entre la pratique et la recherche; celle-ci devant déboucher sur des études qui s'organisent différemment selon des approches linguistiques ou didactiques.

\section{Sous-thème II}

Le sous-thème II a eté consacré aux nouveaux besoins, nouvelles pédagogies. Les travaux en atelier ont montré la convergence des préoccupations, en particulier sur les points suivants :

- Objet de l'enseignement : il faut arriver à dépasser les clivages entre enseignement général et enseignement spécialisé, enseignement à dominante littéraire et enseignement à dominante linguistique; le besoin d'une plus grande pluridisciplinarité entre les départements, voire les facultés a été affirmé. Du côté des langues de spécialité, on a noté le désir de travailler davantage sur les connaissances de base pour assurer une transférabilité d'un domaine à l'autre. Les participants souhaitent bénéficier de recherches dans cet esprit.

- Publics visés : il ne faut pas négliger les publics potentiels (adultes en formation continue), ce qui suppose que l'institution universitaire sache s'adapter aux besoins différents de ces publics professionnels. La transversalité doit demeurer le terme clé. Il conviendrait d'engager une recherche permettant d'analyser ces besoins, et donc d'en inférer des méthodes et des programmes d'enseignement.

- Enseignants: c'est dès la formation initiale de l'enseignant que l'accent devrait être mis non seulement sur les aspects didactiques de son futur métier mais encore sur la nécessaire polyvalence 
qu'il devra manifester. Selon la formule célèbre, «le professeur de langue (maternelle ou étrangère) est professeur de vie: il est celui qui doit apprendre à être». Qu'il soit donc préparé à affronter les situations d'enseignement diversifiées. Une telle exigence suppose des dispositions solides de formation continue et d'échanges d'expériences.

- Outils pédagogiques: on a souvent constaté l'inadaptation des matériels disponibles à la diversité des situations locales; ce qui sous-entend le besoin de produire des outils contextualisés. De tels outils ne peuvent être produits que sur place, mais ils doivent pouvoir bénéficier, dans leur élaboration, de la comparaison avec des expériences analogues.

S'agissant des technologies, il conviendrait d'en venir à une conception plus large des outils utilisables, et de ne pas dédaigner le recours à des technologies qui pour êtrc plus anciennes n'en sont pas moins souvent mieux adaptées aux réalités locales.

\section{à l'AUPELF:}

Les recommandations suivantes sont adressées

- Création d'un réseau intitulé Sociolinguistique. didactique des langues et gestion de la formation. L'idée, là, est d'analyser la variété des usages de la langue française et d'en tirer des conclusions méthodologiques.

- Mise en place d'une structure d'information et d'échanges d'expériences.

- Mesures concrètes en matière de formation des enseignants (stages transrégionaux ou multinationaux, stages d'enseignants dans d'autres universités).

\section{Sous-thème III}

La réflexion a porté sur Langue française et contacts de cultures. Les discussions ont particulièrement mis en lumière les points suivants :

Relation du français et des autres langues

\section{Après avoir constaté}

- que la pluralité linguistique est une constante dans la plupart des situations dans lesquelles le français se trouve impliqué, soit à titre de langue étrangère, soit à titre de langue seconde,

- et que cette diversité linguistique, diversement vécue, ne fait pas partout l'objet d'un programme d'aménagement linguistique clairement engagé,

les participants proposent que l'Université joue un rôle important dans ce processus d'aménagement linguistique. Il conviendrait notamment :

a) d'engager des programmes de recherche et d'enseignement allant dans le sens d'une véritable coopération interculturelle et interlinguistique :

b) de concevoir de nouvelles stratégies tournées vers les champs privilégiés de l'interculturel (études comparatives, traductions, etc.) et d'y adapter la formation des enseignants de français.
Interculturel

On s'est demandé s'il ne convenait pas de commencer la formation-comparaison interculturelle dès le niveau secondaire. Et on a souligné la nécessité de refuser les approximations et l'amateurisme dans le domaine des iravaux interculturels. Les bonnes réponses ne peuvent venir que de l'interdisciplinarité.

On propose donc de favoriser les échanges de professeurs entre espaces francophone et non francophone, d'encourager la création d'équipes multiculturelles pour améliorer la représentation de l'ensemble des pays francophones dans les outils méthodologiques de l'enseignement du français. On propose d'élaborer une typologie des situations d'enseignement prenant en compte les variables culturelles (non systématiquement superposables aux variations sociolinguistiques).

II conviendrait que l'AUPELF s'interroge dans l'organisation des ses rencontres sur l'articulation entre les industries de la langue, la technologie et la dimension culturelle (formation de la personne fonction de l'enseignement dans la saciété). Il serait aussi souhaitable que l'AUPELF suscite, par exemple sous forme de rencontre scientifique de haut niveau, une réflexion approfondie sur les conditions de description des cultures.

Enseignement des littératures et cultures des pays francophones

La réflexion a souligné que l'enseignement de la francophonie se heurte partout à des difficultés nombreuses:

- crédibilité scientifique et légitimation institutionnelle,

- poids symbolique de la France hexagonale,

- statut ambigu des littératures francophones,

- difficulté d'accès aux ceuvres et à la documentation, etc.

Les participants recommandent vivement d'intégrer les enseignements concernant les réalités littéraires et culturelles francophones à tous les niveaux des cursus universitaires d'enseignement du français, et particulièrement en France.

D'autre part, ils suggèrent d'encourager la création sur les médias modernes (télévision, vidéo, etc.) et plus généralement d'orchestrer et de valider le travail d'enseignement et de recherche sur d'autres supports que l'écrit.

Les recommandations suivantes, très concrètes, ont été avancées :

- mise à la disposition des centres d'études francophones, et particulièrement dans les pays non francophones, de bibliothèques et vidéothèques minimales;

- élaboration d'appareils critiques et documentaires accompagnant les éditions à grande diffusion des textes francophones;

- fabrication d'usuels (dictionnaires et grammaires) prenant en compte les réalités linguistiques et culturelles de la francophonie :

- élaboration de banques de données (y compris des répertoires des thèses et des chercheurs et un inventaire des produits médiatiques francophones suscep- 
tibles d'être exploités dans l'enseignement du français);

i encouragement aux échanges scientifiques par l'organisation de séminaires intensifs, colloques, stages de formation des formateurs, particulièrement pour donner une impulsion à la création de nouveaux centres d'études francophones.

\section{Échanges}

Sur la base d'un bilan des actions de coopération interuniversitaire et dans un souci prospectif, les participants ont examiné les stratégies à mettre en œuvre pour définir une nouvelle politique des échanges, qui permettrait de dynamiser le réseau des départements d'études françaises.

Ces échanges doivent équilibrer les enjeux complémentaires du développement des savoir-faire techniques et scientifiques et de la sauvegarde des identités, des savoir-être et des productions culturelles.

Ceci implique à la fois le maintien d'une coopération régionale et l'émergence de flux d'échanges transrégionaux.

Les objectifs suivants ont été particulièrement recommandés :

- la mise à niveau linguistique et scientifique des formateurs, des enseignants et des chercheurs au Nord et au Sud dans des instituts habilités à délivrer des diplômes élémentaires de langue française et des diplômes approfondis de langue française ;

- le perfectionnement de la formation initiale et continue, le recyclage et la spécialisation professionnelle par des stages et des missions dans des instituts étrangers, universités, instituts, entreprises publiques ou privées;

- la mise à disposition de bourses d'excellence prioritairement destinées aux $3^{\mathrm{e}}$ cycles mais aussi à des enseignants en année sabbatique;

- l'interrelation aux niveaux national, régional et transrégional des secteurs de l'enseignement, de la recherche et de la formation, notamment en vue de l'édition et de la diffusion;

- la promotion de la recherche et de la formation en communication et en traduction pour une approche sémiologique contrastive ; l'appui à la documentation bibliographique appropriée et au stockage en banques de données ;

- le développement d'échanges triangulaires où l'AUPELF-UREF assurerait la participation des universitaires francophones et non francophones dans le cadre d'échanges déjà institués sur des bases bilatérales ou dans un cadre coopératif plus large tel que le projet ERAMUS de la Communauté européenne pour la région méditerrannéenne;

- un appui aux centres non francophones chargés de la mise à niveau de personnels amenés à travailler en zone francophone;

- la poursuite des échanges inter-comités dans les domaines ayant rapport au développement et au transfert de technologies ;

- une meilleure intégration des département d'études françaises dans les programmes de l'UREF.

\section{Sous-thème IV}

Le quatrième sous-thème, consacré aux «industries de langue», a été ouvert en séance plénière par des exposés mettant l'accent sur les activités couvertes par les industries de la langue et leur pertinence quant aux problèmes d'enseignement. On y a insisté sur les aspects liés à la documentation en langue naturelle. $\mathrm{La}$ quasi-totalité des textes sont produits sur support informatique (livres, journaux, revues, correspondances administrative et commerciale, etc.). Les textes sont archivés et des méthodes de recherche automatique d'information dans ces textes sont en cours d'élaboration.

L'une des premières conditions mises pour le stockage en vue du traitement automatique est la correction orthographique des textes. Le problème de la représentation orthographique se pose de plusieurs façon en informatique :

- au niveau des codes, et donc des claviers d'ordinateur,

- au niveau de la norme des textes : l'ordinateur bute sur la moindre déviation.

Ces questions techniques rejoignent le problème d'actualité de la réforme orthographique demandée par les enseignants: la difficulté de l'orthographe française est un obstacle à son enseignement comme première et comme seconde langue.

Le second rapport a indiqué que les problèmes que pose la construction des premiers outils de base d'une langue peuvent bénéficier de l'informatique, mais à un niveau simple. Toutes les techniques ambitieuses développées dans les laboratoires ne sont pas pertinentes aux travaux de fondation.

Des présentations plus spécifiques ont été faites en ateliers. On y a souligné la distance qui sépare les recherches et leurs applications. On y a montré la complexité qu'il y avait à accéder à l'information des banques de données. Aussi faudrait-il mettre en garde les utilisateurs naïfs.

Un exposé a traité de la structure formelle des textes, question théorique qui a une application directe dans l'enseignement du style.

On a présenté un logiciel expérimental d'analyse du discours qui donne une bonne idée des outils de traitement dont on disposera à terme.

Des dictionnaires électroniques de divers types, qui ont donné lieu à des démonstrations sur microordinateurs, ont été également présentés.

JEAN-CLAUDE GÉMAR Université de Montréal, Montréal, Canada 